\title{
TÉRMINOS PEYORATIVOS DE GRUPO, ESTEREOTIPOS Y ACTOS DE HABLA
}

\author{
ELEONORA ORLANDO \\ IIF-SADAF-CONICET \\ Universidad de Buenos Aires \\ eorlando0@icloud.com \\ ANDRÉS SAAB \\ IIF-SADAF-CONICET \\ Universidad de Buenos Aires \\ al_saab75@yahoo.com.ar
}

RESUMEN: Este trabajo trata de los términos peyorativos de grupo, es decir, expresiones que se usan paradigmáticamente para hablar de manera despectiva acerca de ciertos grupos identificados en virtud de su origen o descendencia ("sudaca"), raza o nivel social ("negro"), orientación sexual ("maricón"), religión ("moishe"), ideología política ("zurdo"), modo de vida ("puta"), etc. Nos proponemos tanto (i) explicar el significado expresivo de este tipo de términos mediante una versión de la semántica de estereotipos como (ii) analizar sus usos originales y paradigmáticos, aquellos en los que funcionan como insultos, en el marco de una teoría de los actos de habla.

PALABRAS CLAVE: lenguaje expresivo, significado expresivo, conceptos densos, intención peyorativa, fuerza ilocucionaria insultiva

SUMMARY: This paper is about group slurs, i.e., expressions that are prima facie associated with the speaker's conveyance of a derogatory feeling for a certain group of people identified in terms of their origin or descent ("spic"), race ("nigger"), sexual orientation ("faggot"), ethnicity or religion ("kike"), political ideology ("snowflake"), habits or ways of living ("whore"), etc. Our purpose is twofold: (i) explaining the non-truth-conditional or expressive meaning dimension in terms of a version of stereotype semantics, and (ii) applying a speech act theory to the original and paradigmatic derogatory uses of such terms.

KEY WORDS: expressive language, expressive meaning, thick concepts, pejorative intention, insultive illocutionary force

Este trabajo trata de los términos peyorativos de grupo, es decir, expresiones que se usan paradigmáticamente para hablar de manera despectiva acerca de ciertos grupos identificados en virtud de su origen o descendencia ("sudaca"), raza o nivel social ("negro"), orientación sexual ("maricón"), religión ("moishe"), ideología política ("zurdo"), modo de vida ("puta"), etc. Nos proponemos tanto explicar el significado expresivo de este tipo de términos mediante una versión de la semántica de estereotipos como analizar sus usos originales y 
paradigmáticos, aquellos en los que funcionan como insultos, en el marco de una teoría de los actos de habla.

En la primera parte del trabajo, desarrollamos nuestra propuesta semántica: consideramos que los estereotipos culturales asociados con ciertos grupos dan lugar a estereotipos semánticos que constituyen los significados expresivos de los términos peyorativos de grupo correspondientes. Por su naturaleza expresiva, estos significados contribuyen a determinar no las condiciones de verdad sino las condiciones de corrección expresiva de las emisiones en las que aparecen (es decir, no son representacionales o condicional-veritativos). Consideramos que una de sus características fundamentales es su carácter evaluativo: no sólo incluyen conceptos densos sino que también codifican un valor negativo global, esto es, una valencia negativa.

En la segunda parte, complementamos la teoría semántica propuesta con una explicación pragmática de los usos en los que los peyorativos de grupo funcionan como insultos. Defendemos la tesis de que éstos pueden concebirse como un subconjunto de los actos de habla insultivos, esto es, un tipo de acto de habla con una fuerza ilocucionaria especial que denominamos con el neologismo "fuerza insultiva". Desde nuestra perspectiva, el análisis de los usos insultivos tiene interés porque agrega un factor expresivo de índole pragmática, la intención peyorativa, al factor expresivo de índole semántica, el estereotipo, analizado en la primera parte. La intención peyorativa no es semántica sino pragmática porque no es parte constitutiva del estereotipo semántico sino que es un factor aportado por ciertos usos especiales (los insultivos) de los peyorativos de grupo. Por eso mismo, su análisis se lleva a cabo en el marco de algunas reflexiones pragmáticas clásicas en torno a la noción de fuerza ilocucionaria. ${ }^{1}$

\section{Una semántica de estereotipos para los términos peyorativos de grupo}

\subsection{El marco general de las semánticas mixtas}

Una primera característica a destacar es el hecho de que los términos peyorativos de grupo, como los antes mencionados "sudaca", "maricón", "moishe", tienen contrapartes neutras, "sudamericano", "homosexual", "judío", respectivamente — con las cuales están en

${ }^{1}$ Queremos destacar que todas las apariciones de los términos peyorativos de grupo que se encuentran en este texto son menciones y no usos. Aun aclarado esto, no está de más dejar en claro que no suscribimos el valor negativo que estos términos involucran. 
competencia - es decir, ambos tipos de términos forman pares que parecen enfrentar a los hablantes competentes a la posibilidad de una elección voluntaria. Pero, a diferencia de sus contrapartes neutras, los peyorativos son términos mixtos, es decir, prima facie tienen no sólo una dimensión descriptiva sino también una dimensión expresiva. La propuesta que vamos a desarrollar se alinea con aquellas semánticas mixtas o dualistas que se inspiran en Kaplan (1999), según las cuales: (i) esas dos dimensiones son semánticamente independientes entre sí y, por tanto, deben ser explicadas de manera diferenciada; (ii) el significado descriptivo de un peyorativo de grupo es idéntico al significado descriptivo de su contraparte neutra (Tesis de la Identidad). ${ }^{2,3}$ En este trabajo no argumentaremos en favor de estas tesis sino que las daremos por sentadas - como es sabido, uno de los principales argumentos en favor de (i) se basa en el hecho de que el significado expresivo de los peyorativos no es afectado por operadores que afectan las condiciones de verdad de una emisión, tales como la negación, la condicionalización, los verbos de actitud proposicional, etc., es decir, tiene alcance por fuera de tales operadores. ${ }^{4}$

$\mathrm{Al}$ igual que estas teorías, consideramos que el significado descriptivo puede explicarse en términos de la noción de representación, entendida ya sea intensionalmente (como una intensión, es decir, en el caso particular de los términos generales, una propiedad) o de manera puramente extensional (como un conjunto de individuos); de este modo, el significado descriptivo será concebido como un significado representacional o condicional-veritativo (truth-conditional), esto es, como aquello que el término contribuye a las condiciones de

${ }^{2}$ Véanse, por ejemplo, los desarrollos formales que McCready (2010), Predelli (2013) y Gutzmann (2015) proponen. Un trabajo pionero en la formalización de la semántica de los expresivos se encuentra en la obra de Potts $(2003,2005)$.

${ }^{3} \mathrm{La}$ Tesis de la Identidad está en el centro de las Teorías de la Contraparte Neutra, tales como las de Jeshion (2013a, 2013b), Predelli (2013), Whiting (2013), entre otras. Una defensa general de la tesis en cuestión puede encontrarse en Caso y Lo Guercio (2016). Para posturas contrarias, véanse Hom $(2008,2010)$ y Losada (2017).

${ }^{4}$ Véanse, por ejemplo, Potts (2005), para una discusión detallada sobre esta propiedad, y McCready (2010) para su aplicación a las expresiones mixtas que tratamos en este trabajo. Ambos autores consideran que se trata de una característica fundamental de las implicaturas convencionales. Otros autores, como Schlenker (2007), consideran, en cambio, que se trata de un efecto del carácter presuposicional de los expresivos y los términos mixtos. Si bien acordamos con que los términos peyorativos de grupo cumplen con esta propiedad de manera general, no suscribimos que se trate de implicaturas convencionales o de presuposiciones. 
verdad de las emisiones en las que aparece. ${ }^{5}$ En cuanto al significado expresivo, a diferencia de otras teorías dualistas (que apelan a nociones tales como las de implicatura convencional, sesgo y regla de uso), propondremos una explicación semántica que asignará un papel central a la noción de estereotipo.

Es oportuno aclarar entonces que el significado expresivo no está constituido por un sentimiento o estado puramente afectivo, como podría pensarse que ocurre en el caso de las interjecciones, tales como, por ejemplo, "ay", cuyo significado se agota en cierto sentimiento o estado de dolor. En el caso de los términos peyorativos de grupo, como se verá a continuación, concebiremos al significado expresivo como una entidad compleja: el mencionado estereotipo, que incluye a su vez un componente mixto cognoscitivo-evaluativo, constituido por un conjunto de conceptos (fundamentalmente, conceptos densos), y un componente puramente evaluativo, constituido por una valencia negativa (que determinará sistemáticamente una actitud evaluativa negativa por parte del hablante competente).

Asimismo, consideraremos que el estereotipo, en su papel de significado expresivo, cumple con la función que Kaplan (1999) asignó a los significados expresivos en general (como sea que fueren concebidos): imponer restricciones al uso de los términos correspondientes. Mientras que un término puramente descriptivo o expresivamente neutro puede usarse en todo contexto (sin restricciones), un término que posee un significado expresivo sólo puede usarse en algunos contextos - por ejemplo, hay contextos en los que no es expresivamente correcto usar "sudaca" o "maricón". Como es claro, el concepto de corrección expresiva no tiene ningún matiz ético o moral: los contextos en los que el uso de términos peyorativos de grupo resulta expresivamente correcto no son necesariamente contextos en los que tal uso es moralmente correcto (sino, como se verá más adelante, todo lo contrario). De este modo, el significado expresivo de un término cumple con la tarea de seleccionar aquellos contextos en los que la emisión que lo contiene resulta (no verdadera sino) expresivamente correcta; dicho de otro modo, es su contribución a las condiciones (no de verdad sino) de corrección expresiva de la emisión involucrada.

En síntesis, en la línea de las teorías mixtas o dualistas, diremos que los términos peyorativos de grupo requieren una semántica

\footnotetext{
${ }^{5}$ En relación con la dimensión representacional o condicional-veritativa, no nos pronunciamos en favor de ninguna teoría semántica particular, esto es, dejamos abierta la elección entre una teoría intensionalista (que apela a mundos posibles) o una extensionalista (que apela a hechos o condiciones de verdad efectivas).
} 
comprometida con dos dimensiones del significado, independientes entre sí: (i) un significado representacional o condicional-veritativo (truth-conditional), que contribuye a determinar las condiciones de verdad de las emisiones en las que aparecen; y (ii) un significado expresivo (non-truth-conditional o, en la terminología de Gutzmann (2015), use-conditional) que contribuye a determinar sus condiciones de corrección expresiva.

En términos de uno de los ejemplos anteriores, "sudaca" tiene, por un lado, un significado representacional, la propiedad de ser sudamericano (en un marco intensionalista) o el conjunto de los sudamericanos (en uno extensionalista), y, por otro, un significado expresivo, que deberá especificarse en detalle pero que en principio está relacionado con el hecho de que "sudaca" sólo puede usarse en algunos contextos. Su contraparte neutra, en cambio, "sudamericano", tiene su mismo significado representacional (representa la propiedad de ser sudamericano o el conjunto de los sudamericanos), pero no tiene significado expresivo, por lo que puede usarse en todo tipo de contexto, sin ninguna restricción. De este modo, por un lado, las emisiones de (1) y su contraparte neutra (2)

(1) Diego es sudaca.

(2) Diego es sudamericano.

tienen la misma condición de verdad:

(3) "Diego es sudaca" es verdadera si y sólo si Diego nació en América del Sur.

(4) "Diego es sudamericano" es verdadera si y sólo si Diego nació en América del Sur.

Pero, por otro lado, toda emisión de (1), a diferencia de las emisiones de (2), tendrá también una condición de corrección expresiva, que hace referencia al conjunto de contextos en los que resulta expresivamente correcta: como dijimos anteriormente, es en relación con la selección de tal conjunto que la noción de estereotipo desempeña, desde nuestro punto de vista, un papel explicativo central.

1.2. De los estereotipos culturales a los estereotipos semánticos

Como es sabido, a lo largo de la historia de las comunidades humanas, la existencia de actitudes y prácticas discriminatorias de diversos tipos ha dado lugar a prejuicios, fuertemente atrincherados, 
según los cuales ciertas personas, en virtud de su origen, raza, género, orientación sexual, etc. son consideradas inferiores al resto y, por tanto, resultan excluidas del desempeño de ciertas funciones y confinadas a realizar otras, también consideradas inferiores. Tales prejuicios reflejan entonces una historia de actitudes individuales y prácticas sociales discriminatorias profundamente injustas. Constituyen estereotipos culturales, esto es, conjuntos de creencias genéricas, pobres, esquemáticas, que hacen referencia a diversos rasgos asociados con determinada raza, género, preferencia sexual, etc., a los que se asigna en conjunto un valor global negativo. El prejuicio que estructura un estereotipo cultural asociado con determinado grupo de personas involucra un conjunto de rasgos con carácter evaluativo que determinada comunidad atribuye paradigmáticamente a ese grupo, pero está claro que hay miembros del grupo que no tienen ninguno de ellos y que alguien puede tenerlos y no pertenecer al grupo.

Ahora bien, entre las prácticas sociales de la opresión, las comunidades humanas cuentan con diversos mecanismos simbólicos que sirven a los fines de la discriminación, marginación y caricaturización de las personas; en particular, aspectos como los antes mencionados, esto es, el origen, la raza, el género, la orientación sexual han inducido e inducen distintas formas de violencia simbólica, entre las cuales se encuentra el uso de los términos peyorativos de grupo. ${ }^{6}$ Nuestra idea es entonces derivar a partir del estereotipo cultural asociado con cierto grupo de personas un estereotipo semántico vinculado convencionalmente con el término peyorativo para ese grupo. Retomando el ejemplo anterior, el hecho de que en determinada comunidad exista un estereotipo cultural asociado con los sudamericanos fundamenta la adscripción de un estereotipo semántico al término peyorativo para ese grupo, "sudaca". Como se destacó anteriormente, la manera neutra de hacer referencia a ese mismo grupo, "sudamericano", no tiene una dimensión semántica expresiva: es una expresión puramente descriptiva y, en tanto tal, posee sólo una dimensión representacional (constituida por una intensión y/o una extensión). La función del estereotipo semántico asignado a un peyorativo de grupo es entonces

\footnotetext{
${ }^{6}$ Esto hace a los peyorativos de grupo fuertemente relativos a las distintas comunidades lingüísticas y periodos históricos — por más vagas que sean las expresiones "comunidad lingüística" y "periodo histórico"—. Por ejemplo, "sudaca" se usa actualmente en España y América Latina para discriminar a los sudamericanos; en inglés "nigger" no es semánticamente equivalente a "negro" en todas las comunidades de habla hispana, dado que es un término peyorativo racial en Estados Unidos, pero social en países como Argentina; "queer" pasó de ser en Estados Unidos un término peyorativo a ser un término neutro para designar a los homosexuales.
} 
contribuir a la dimensión semántica expresiva que éste, a diferencia de su contraparte neutra, posee.

En líneas generales, consideramos que un estereotipo es un concepto complejo, constituido por conceptos más simples. ${ }^{7}$ ¿De qué tipo de concepto se trata? Como la palabra lo indica, se trata de un concepto estereotípico o prototípico, cuya naturaleza está inspirada en las reflexiones de Wittgenstein (1953) en torno al significado de los términos del lenguaje natural. Como se recordará, desde la perspectiva wittgensteiniana, un término significa una lista de características "que se solapan y se entrecruzan", ninguna de las cuales precisa estar presente para que algo cuente como una instancia correcta de aplicación del término; cada instancia podrá tener un subconjunto distinto de las características de la lista - es decir, ninguna característica involucra una condición necesaria para aplicar el término a un objeto- - Conversamente, un objeto puede tener la mayor parte de las características incluidas en la lista y, sin embargo, no ser una instancia correcta de aplicación del término - esto es, la presencia del conjunto de características no es una condición suficiente para aplicar el término a un objeto-. Puede recordarse cómo esta idea se aplica al término "lenguaje":

En vez de indicar algo que sea común a todo lo que llamamos lenguaje, digo que no hay nada en absoluto común a estos fenómenos por lo cual empleamos la misma palabra para todos — sino que están emparentados entre sí de muchas maneras diferentes. Y a causa de este parentesco, o de estos parentescos, los llamamos a todos "lenguaje". (Wittgenstein 1953, p. 87, \#65)

Y más adelante, después del conocido parágrafo en el que analiza el término "juego", Wittgenstein concluye que la mejor manera de dar cuenta del significado de un término es apelar a la noción metafórica de parecidos de familia:

Y el resultado de este examen reza así: vemos una complicada red de parecidos que se superponen y entrecruzan. Parecidos a gran escala y en detalle. [...] No puedo caracterizar mejor esos parecidos que con la expresión "parecidos de familia"; pues es así como se superponen y entrecruzan los diversos parecidos que se dan entre los miembros de una

${ }^{7}$ Es posible considerar que el estereotipo incluye, además de conceptos, imágenes o representaciones icónicas típicas. Pero dejaremos este aspecto de lado para no complicar la exposición. 
familia: estatura, facciones, color de los ojos, andares, temperamento, etc. -Y diré: los "juegos" componen una familia. (p. 89)

Nos interesa destacar no sólo que no hay nada esencial, un conjunto de condiciones necesarias y suficientes para aplicar el término a un objeto determinado, sino, además, que la lista de características está abierta: puede haber rasgos nuevos (en el sentido de no haber estado presentes en aplicaciones anteriores) que sean relevantes para la aplicación del término a un caso particular. Ahora bien, esta concepción del significado de un término como dado por una lista abierta de características fundamenta la idea de concepto estereotípico o prototípico asociada al término en cuestión: se trata de un concepto complejo integrado por una lista abierta de conceptos más simples, que no codifica condiciones individualmente necesarias y conjuntamente suficientes para la aplicación del concepto a un objeto.

Como también se recordará, la teoría semántica que Putnam (1970, 1975) propone hace un uso restringido de esta noción de concepto prototípico. En primer lugar, la aplica sólo a un subconjunto de las expresiones del lenguaje: los términos de clases naturales. En segundo lugar, el concepto prototípico, lo que Putnam llama "estereotipo", no agota el significado de tales términos sino que constituye uno de sus factores fundamentales, junto a la extensión. De acuerdo con Putnam, todo término de clase natural (tal como, por ejemplo, "tigre") tiene un significado dual constituido fundamentalmente por el estereotipo asociado y la extensión. Mientras que la extensión es la clase natural que está en el mundo externo (la especie Panthera tigris), el estereotipo está formado por un conjunto de características típicas o paradigmáticas que proporcionan una idea convencional, general, imprecisa, aproximada e ingenua de algo, a saber, el conjunto de ejemplares (o, si se trata de un término general de masa, muestras) particulares de la clase natural en cuestión. Por ejemplo, en relación con "tigre", sostiene:

hay una teoría de algún modo asociada con la palabra "tigre"; no la teoría que de hecho creemos acerca de los tigres, la cual es muy compleja, sino una teoría excesivamente simplificada que describe, para decirlo de algún modo, un estereotipo de tigre. Describe [...] un miembro normal del género natural. (Putnam 1970, p. 148; la traducción es nuestra.)

De este modo, el estereotipo de "tigre" puede especificarse por medio de una lista de características como la siguiente: 


\section{"tigre" = CON RAYAS TRANSVERSALES NEGRAS Y ANARANJADAS, DE CUATRO PATAS, HABITANTE DE LA SELVA ${ }^{8}$}

En la medida en que se trata de un concepto prototípico, el estereotipo no codifica un conjunto de condiciones individualmente necesarias y conjuntamente suficientes para que un individuo pertenezca a la extensión del término correspondiente. En otras palabras, la extensión de un término de clase natural no está determinada por el estereotipo, pues el estereotipo y la extensión son dos componentes semánticos independientes entre sí - en este aspecto el estereotipo es distinto del sentido fregeano y de la noción tradicional de intensión, ambos determinantes de la extensión-. ${ }^{9}$ Putnam aclara explícitamente que el término de clase natural obtiene (y mantiene) su extensión no mediante la satisfacción de una definición asociada sino mediante un mecanismo histórico-causal: el término queda originalmente fundado o anclado en una clase natural en un momento histórico determinado, y esta conexión se extiende en el tiempo a través de una cadena de comunicación. De este modo, en términos del ejemplo anterior, un individuo no necesita tener rayas transversales negras y anaranjadas, ni ninguno de los otros rasgos incluidos en el estereotipo, para ser un ejemplar de la especie Panthera tigris, es decir, para ser un tigre (hay tigres con rayas grises y blancas, tigres de tres patas y tigres que habitan en zoológicos urbanos), y puede haber individuos que tengan rayas transversales negras y anaranjadas, cuatro patas y habiten en la selva, y no sean tigres (sino que sean, por ejemplo, pumas pintados o robots con aspecto de tigres). Además, el estereotipo puede contener "desinformación" o "información falsa" acerca de los individuos a los que se aplica correctamente el término. Por lo tanto, el hecho de que cierto rasgo, por ejemplo, CON RAYAS TRANSVERSALES NEGRAS Y ANARANJADAS, esté incluido en el estereotipo de "tigre" no implica que la emisión de

(5) Los tigres tienen rayas transversales negras y anaranjadas.

\footnotetext{
${ }^{8}$ Las mayúsculas son la convención elegida para designar conceptos.
}

${ }^{9} \mathrm{Un}$ aspecto en el que el estereotipo sí se asemeja al sentido fregeano y a la noción tradicional de intensión está relacionado con el hecho de que, al igual que éstos, constituye una regla convencional para usar competentemente un término. Según Putnam (1975), el estereotipo contiene la información y la desinformación que es necesario adquirir para aprender la palabra: el hablante está obligado a captar los rasgos incluidos en el estereotipo asociado si quiere ser competente con la palabra en cuestión (noción putnamiana de obligación lingüística). Véase más adelante de qué manera proponemos moderar esta obligación en el caso de los estereotipos de los términos peyorativos de grupo. 
sea verdadera - y, muchos menos, que sea una verdad analítica o verdadera en virtud del significado de "tigre"-. Lo que nos interesa destacar es que, en la medida en que el estereotipo no tiene la función semántica de determinar la extensión del término, no es un significado representacional o condicional-veritativo en el sentido antes señalado: no es parte de la contribución del término a la determinación de las condiciones de verdad de las emisiones en las que aparece.

Ahora bien, nuestra propuesta es asignar a los términos peyorativos de grupo un estereotipo semántico concebido en términos de un concepto prototípico con las características puestas de relieve en los parágrafos anteriores. Se trata de un concepto complejo constituido por un conjunto de conceptos más simples, cada uno de los cuales expresa un rasgo del estereotipo cultural asociado con el grupo correspondiente. A modo de ejemplo, el término peyorativo de grupo "sudaca" posee un estereotipo semántico, derivado del estereotipo cultural asociado con los sudamericanos, que podría describirse en parte como sigue:

\section{"sudaca" = ILEGAL, TENDIENTE A TENER TRABAJOS TEMPORARIOS, DE ASPECTO INFORMAL, CON UN ACENTO PECULIAR, CON RASGOS INDÍGENAS, etc.}

Algunos de estos rasgos son centrales (ILEGAL, TENDIENTE A TENER TRABAJOS TEMPORARIOS, DE ASPECTO INFORMAL, CON UN ACENTO PECULIAR) mientras que otros son tal vez más periféricos (CON RASGOS INDÍGENAS). Hay distintos aspectos que queremos aclarar y enfatizar.

En primer lugar, a diferencia de lo que ocurre en la teoría putnamiana de los términos de clase natural, el estereotipo de un término peyorativo no constituye su intensión sino lo que antes caracterizamos como su significado expresivo. Para nosotros, tanto la intensión como la extensión de un término constituyen su significado representacional o condicional-veritativo, es decir, aquello que el término aporta a las condiciones de verdad de las emisiones en las que aparece. En la medida en que el estereotipo no cumple ese papel, no es un componente del significado representacional del término peyorativo. Como mencionamos anteriormente, un peyorativo de grupo como "sudaca", al ser mixto, posee, por un lado, un significado representacional constituido por una intensión y/o una extensión (idénticas a las de su contraparte expresivamente neutra, "sudame- 
ricano"); por otro lado, un significado expresivo constituido por el estereotipo especificado anteriormente. ${ }^{10}$

En segundo lugar, dada la independencia señalada entre ambos factores semánticos, el hecho de que un determinado rasgo esté incluido en el estereotipo de un peyorativo de grupo no implica que sea verdad (ni mucho menos, una verdad analítica) que todos los individuos que tienen la propiedad representada por el término o que pertenecen a su extensión posean de hecho ese rasgo. Por lo tanto, el hecho de que ILEGAL esté incluido en el estereotipo de "sudaca" no implica que la emisión de

(6) Los sudacas son ilegales.

sea verdadera (ni, mucho menos, analíticamente verdadera, es decir, verdadera en virtud del significado de "sudaca"): alguien puede ser sudamericano (es decir, tener la propiedad representada por "sudaca" o pertenecer a la extensión del término) y ser legal. Más aún, también en paralelismo con los términos de clase natural, alguien puede no tener ninguno de los rasgos del estereotipo semántico de "sudaca" y ser sudamericano (hay sudamericanos que son legales, tienen trabajos permanentes, etc.) o tener gran parte de esos rasgos y no serlo (ser, por ejemplo, mexicano o cubano).

En tercer lugar, al igual que en la teoría de Putnam, el estereotipo está relacionado con la competencia lingüistica: para ser competente en el uso del peyorativo de grupo el hablante está obligado a conocer el estereotipo asociado. Sin embargo, sobre la base de la idea wittgensteiniana, según la cual un término se define en términos de una lista abierta, consideramos que no hay un conjunto determinado de rasgos que todo hablante esté obligado a captar para usar un peyorativo de grupo de manera competente: su obligación lingüística es conocer cualquier subconjunto de rasgos perteneciente a una lista abierta. Como consecuencia, si bien es altamente probable que la mayoría de los hablantes competentes conozcan los rasgos centrales del estereotipo, distintos hablantes pueden captar distintos aspectos o partes: para ser competente no se requiere acceder a la totalidad del estereotipo, lo que es, por lo demás, imposible, dado que está constituido por una lista abierta de conceptos. Además, tal captación

${ }^{10}$ De este modo, según nuestra propuesta, la propiedad de ser sudamericano, esto es, la de pertenecer al colectivo estereotipado, no es de ningún modo una de las propiedades expresadas por el estereotipo asociado sino la propiedad representada por "sudaca"; dicho de otro modo, esa propiedad no da lugar a un rasgo del estereotipo asociado sino que constituye la extensión del peyorativo. 
no implica que el hablante competente deba creer que todos, la mayoría, los miembros normales o siquiera algunos miembros del grupo poseen de hecho los rasgos en cuestión: sólo implica saber que el término peyorativo correspondiente está convencionalmente asociado con esos rasgos.

Asimismo, como ocurre, según Putnam, con los términos de clase natural, el hablante competente puede ser ignorante o estar equivocado acerca de la extensión del peyorativo. Un hablante puede no saber exactamente qué especie representa "tigre" o creer equivocadamente que el término se aplica tanto a los tigres como a los leopardos sin por ello no ser competente en su uso: para la competencia basta con ser capaz de asociar con "tigre" el estereotipo correspondiente. ${ }^{11}$ Del mismo modo, un hablante puede no saber exactamente qué grupo de personas representa "sudaca" o creer equivocadamente que el término se aplica tanto a los sudamericanos como a los cubanos y a los nacidos en las Islas Canarias y, sin embargo, si es capaz de asociar con el término el estereotipo correspondiente, será competente en el uso de "sudaca". En consecuencia, quien, llevado por la ignorancia o el error, dijera de un leopardo lo expresado por (7)

(7) Ése es un tigre con un pelaje fascinante.

estaría diciendo algo falso, aunque el animal señalado tuviera efectivamente un pelaje fascinante. De la misma manera, si la ignorancia o el error lo llevaran a aplicar "sudaca" a un cubano o, incluso, a un holandés con varios de los rasgos del estereotipo asociado (ILEGAL, TENDIENTE A TENER TRABAJOS TEMPORARIOS, etc.) y emitiera un caso de (8)

(8) Él es un sudaca en problemas.

estaría diciendo algo falso, aunque la persona en cuestión estuviera realmente en problemas. Se trataría, sin embargo, de un hablante perfectamente competente con los términos "tigre" y "sudaca" respectivamente: tanto en el caso de los términos de clase natural como en el de los peyorativos de grupo, la competencia es compatible con

\footnotetext{
${ }^{11}$ Véase específicamente lo dicho por Putnam (1970) en relación con los términos "olmo" y "haya".
} 
la ignorancia y el error acerca de cuál es exactamente la propiedad representada en cada caso. ${ }^{12,13}$

\subsection{El carácter evaluativo del estereotipo semántico}

Hay un aspecto fundamental en el que los estereotipos semánticos de los peyorativos de grupo se distinguen de los de términos de clase natural: a diferencia de estos últimos, los primeros tienen un carácter evaluativo. Y esto se deriva del hecho de que los estereotipos culturales asociados con los grupos correspondientes también lo tienen.

En primer lugar, incluyen, junto a conceptos simples descriptivos, otros que son mixtos, en parte descriptivos y en parte evaluativos. Nuestro ejemplo, el estereotipo de "sudaca", ilustra esta variedad: mientras que CON RASGOS INDÍGENAS es un concepto puramente descriptivo, ILEGAL es un concepto mixto, es decir, es tanto descriptivo como evaluativo. Más específicamente, los conceptos mixtos son llamados "densos" (thick concepts) e incluyen los conceptos éticos, como VALIENTE, CRUEL, BRUTAL, CASTO, etc. y estéticos, como ARMONIOSO, SOMBRÍO, TRÁGICO, PERTURBADOR, etc. De acuerdo con Williams (1985), quien se ocupa sólo de los éticos, se trata de conceptos que sirven tanto para describir a un individuo como para generarnos una actitud en relación con él o guiar nuestra acción en el mundo. Sin pretender profundizar en el debate meta-ético, diremos que un concepto denso no sólo representa una propiedad sino que también codifica un valor global (de signo positivo o negativo), de modo tal que la posesión de dicha propiedad por parte de un individuo será por lo general sistemáticamente evaluada de acuerdo con tal valor — en la línea del debate meta-ético, llamaremos "valencia" al valor codificado en el concepto-. ${ }^{14}$ En términos de un ejemplo, VALIENTE se aplica a una persona que se considera que ha arriesgado su vida razonablemente en beneficio de otros, dando lugar a una situación general que es vista como

\footnotetext{
${ }^{12}$ Agradecemos un comentario de un evaluador anónimo que motivó esta aclaración.

${ }^{13}$ En este aspecto el peyorativo "sudaca" se distingue de su contraparte neutra, "sudamericano": a diferencia de lo que ocurre con el primero, la competencia en el uso del segundo no es compatible con ser ignorante o estar equivocado acerca de la propiedad que representa, esto es, la de haber nacido en América del Sur. La razón de esto es que este último, a diferencia del primero, parece ser un término descriptivo, de modo tal que su extensión es fijada y transmitida descriptivamente (y no histórico-causalmente).

${ }^{14}$ El debate ha enfrentado a cognitivistas (tales como McDowell (1981), Kirchin (2017)) y no cognitivistas (Gibbard (1992), Blackburn (1992), entre otros).
} 
moralmente mejor que la situación previa; de este modo, posee una valencia positiva. En contraste, ILEGAL, concepto denso incluido en el estereotipo de "sudaca", se aplica a alguien que no está autorizado a habitar o trabajar en un determinado país o región, y posee una valencia negativa. Podría decirse entonces que esta clase de estereotipo tiene un componente mixto o cognoscitivo-evaluativo, en tanto el conjunto de conceptos que lo constituye incluye conceptos densos. ${ }^{15}$

En segundo lugar, independientemente de las valencias particulares que posean los distintos conceptos densos (y puramente evaluativos) incluidos en el estereotipo de un peyorativo de grupo, el estereotipo mismo tiene siempre una valencia negativa. Esto revela, desde nuestra perspectiva, que esa valencia no es en realidad el producto de los conceptos evaluativos que lo componen sino que resulta de algo más profundo, i.e., la actitud y la práctica de discriminación, marginación y caricaturización que determina en primer lugar la exclusión del grupo de la esfera de ciertas funciones según la creencia de que se trata de seres inferiores, y que consecuentemente da lugar a las demás creencias prejuiciosas que están en la base del estereotipo cultural. ${ }^{16}$ De este modo, peyorativos de grupo, como "sudaca", "negro", "maricón", "moishe", "puta", tienen una clara significación política. En otras palabras, el valor negativo que codifican sus respectivos estereotipos semánticos es producto de su origen en estereotipos culturales surgidos de actitudes y prácticas de discriminación, marginación y caricaturización que legitiman una estructura social desigual e injusta. ${ }^{17}$ Ahora bien, la valencia negativa constituye un componente evaluativo del estereotipo que es independiente de toda actitud individual: como destacamos anteriormente, indica que el hablante competente típico evaluará paradigmáticamente a los miembros del

${ }^{15}$ Algunos estereotipos pueden incluir también conceptos puramente evaluativos, es decir, sin ningún componente descriptivo, tales como FEO, un concepto estético fino (thin) y MALO, un concepto ético fino: ambos sólo codifican un valor global negativo. Por ejemplo, el estereotipo de "bolita", peyorativo usado en Argentina en relación con los bolivianos, incluye FEO.

${ }^{16}$ Esto es lo que, en nuestra opinión, quiere expresar Tirrell (1999, p. 45) cuando dice que la nota fundamental del estereotipo asociado con "nigger" es la idea de inferioridad o subordinación de aquellos a los que se aplica.

${ }^{17}$ Es oportuno aclarar que hay ejemplos, como "zurdo" ("rojo"), cuya significación política es más directa: proviene del rechazo, por parte de ciertos sectores de la comunidad, de grupos que sustentan ciertas ideologías políticas. Podría pensarse que hay casos excepcionales, como el de "nazi" (término peyorativo derivado del término clasificatorio empleado para designar a los miembros del Partido Nacionalsocialista Obrero Alemán, conocido como Partido Nazi), el rechazo, lejos de dar lugar a una estructura social desigual e injusta, está moralmente justificado. 
grupo correspondiente de acuerdo con tal valencia, independientemente de que alguien en particular lo haga.

Diremos entonces que lo que constituye el significado expresivo de un peyorativo de grupo es un estereotipo que comprende un componente mixto o cognoscitivo-evaluativo (un conjunto de conceptos de distinto tipo, muchos de los cuales son densos) y un componente puramente evaluativo (una valencia negativa).

De este modo, hay dos aspectos que queremos destacar. En primer lugar, ser competente en el uso de un peyorativo de grupo requiere no solo saber que el término está convencionalmente asociado con ciertos rasgos sino también que posee una valencia negativa. Por lo tanto, ser competente en el uso de "sudaca" implica saber no sólo que el término está convencionalmente asociado con los rasgos antes mencionados sino también que posee una valencia negativa.

En segundo lugar, como dijimos al comienzo, el significado expresivo es lo que el término aporta a la determinación de las condiciones de corrección expresiva de las emisiones en las que aparece, esto es, a la selección de aquellos contextos en los que tales emisiones resultan expresivamente correctas. Volviendo al ejemplo, "sudaca" aporta el mencionado estereotipo, de manera tal que la condición de corrección expresiva de una emisión de (1) puede especificarse como sigue:

(9) "Diego es sudaca" es expresivamente correcta si y sólo si es emitida en un contexto en el que está vigente un estereotipo cultural asociado con los sudamericanos, condensado en el estereotipo semántico del término peyorativo utilizado, "sudaca",

de lo que se sigue que emitir (1) en un contexto en el que el mencionado estereotipo cultural no existe resultará expresivamente incorrecto. Como resulta obvio en el ejemplo, la noción de corrección expresiva no involucra ningún sentido ético de "corrección", dado que los contextos en los que está vigente un estereotipo cultural como el mencionado son contextos reprobables desde el punto de vista moral.

\section{La dimensión pragmática de los términos peyorativos de grupo}

2.1. Los distintos tipos de usos

Dadas las consideraciones anteriores, todo uso particular de un término peyorativo de grupo implicará respaldar de alguna manera el estereotipo cultural condensado en el estereotipo semántico o, como 
dijimos antes, re-activar o hacerse eco de las actitudes de discriminación, marginación y caricaturización que están en la base de los prejuicios que fundamentan una estructura social desigual e injusta (con la sola excepción del uso apropiado que se mencionará en breve). Usar un término de ese tipo implicará siempre realizar un acto de habla con significación política - la misma significación política que tienen los términos mismos como entidades lingüísticas-. Ahora bien, hay distintos tipos de usos de los peyorativos de grupo. Por un lado, está el uso irreflexivo, en el que el hablante respalda el estereotipo de manera pasiva, sin tener una actitud despectiva individual, tal como en la emisión de (10)

(10) Envío un cálido saludo de fin de año a todos mis queridos amigos sudacas.

Similar a éste es el uso bromista, entre amigos cercanos, tal como ilustra la emisión de (11)

(11) ¡Nuestro sudaca extraña vivir en el tercer mundo!

Asimismo, está el uso apropiado, en el que el término peyorativo es sistemáticamente usado por los mismos miembros del grupo al que se aplica, muy probablemente en este caso con un respaldo diferente del estereotipo, aparentemente con la intención concomitante de revertir su valencia negativa, como se refleja en el uso de "puto" incluido en la emisión de (12)

(12) Un grupo de travestis, homosexuales y trans decidimos formar la Agrupación Nacional Putos Peronistas. ${ }^{18}$

En cambio, el uso original y paradigmático es aquel mediante el cual el hablante insulta a una persona por pertenecer al grupo de referencia. Desde nuestra perspectiva, en este uso, a diferencia de lo que ocurre en los anteriores, el respaldo del estereotipo es un respaldo activo, caracterizado por la presencia de una actitud emocional por parte del hablante individual que llamaremos intención peyorativa. Como su nombre lo indica, la intención peyorativa involucra desprecio, es decir, una emoción compleja. ${ }^{19}$

\footnotetext{
${ }^{18}$ Un ejemplo característico en inglés es "You are my nigga!" (dicho por un chico afroamericano a otro chico afroamericano).

${ }^{19}$ Entendemos al desprecio como una emoción compleja con tres componentes: uno doxástico, uno evaluativo y uno afectivo.
} 
Proponemos entonces clasificar los usos de los términos peyorativos de grupo en dos tipos principales: por un lado, los usos no insultivos; por otro, los usos insultivos. Nuestra tesis en este punto es que estos últimos, caracterizados por la presencia de una intención peyorativa individual, constituyen un tipo especial de acto de habla o un acto de habla con una fuerza ilocucionaria especial que llamaremos "insultiva". Cabe aclarar que, en la medida en que la fuerza insultiva está caracterizada por la expresión de una emoción (el desprecio), puede considerarse un tipo particular de fuerza expresiva. ${ }^{20}$

Es oportuno notar que es posible realizar un acto de este tipo sin usar términos peyorativos de grupo sino oraciones con términos puramente descriptivos pero con elementos prosódicos que indican la presencia de una intención peyorativa o que están acompañadas por un gesto despectivo, tales como la emisión de (13) realizada con tono despectivo,

(13) No creo que ese sudamericano consiga el trabajo.

También es posible insultar mediante el uso de otro tipo de expresiones peyorativas, tales como el epíteto en (14):

(14) Ese hombre es un completo idiota.

Por consiguiente, los usos insultivos de los términos peyorativos de grupo constituyen un subconjunto dentro del conjunto de los actos de insultar o actos de habla con fuerza ilocucionaria insultiva - los cuales, como dijimos, son a su vez un subconjunto del conjunto más amplio de los actos con fuerza ilocucionaria expresiva-.

Por razones de espacio, no analizaremos los usos no insultivos sino que nos centraremos en los usos insultivos de los peyorativos de grupo. Como mencionamos en la introducción, consideramos que el análisis de los usos insultivos tiene interés porque éstos agregan

${ }^{20}$ Un evaluador anónimo ha señalado que, de acuerdo con Neu (2007), es posible insultar sin tener la intención de hacerlo. Ante todo, cabe aclarar que nosotros, a diferencia de Neu, nos restringimos a la consideración de los usos insultivos de los términos peyorativos de grupo — como aclaramos más adelante en el texto, no nos ocupamos de insultos que involucran el uso de otras expresiones peyorativas-. En segundo lugar, consideramos que los argumentos presentados en 2.2 y 2.3 constituyen buenas razones para creer que insultar, al igual que prometer y advertir, es un tipo de acto de habla, y como tal, una forma de acción intencional. Por eso mismo, nos interesa especialmente hacer una distinción conceptual entre insultar y ofender (véanse las pp. 54 y 55) lo cual sí puede ser considerado una consecuencia perlocucionaria que está más allá del control del hablante, esto es, que no está determinada por sus intenciones. 
un factor expresivo de índole pragmática, la intención peyorativa, al factor expresivo de índole semántica, el estereotipo, analizado en la primera parte. De este modo, el objetivo principal de esta segunda parte es mostrar que es posible identificar un tipo de fuerza ilocucionaria insultiva, característica de los usos insultivos de los peyorativos de grupo, para lo cual presentaremos dos argumentos. El primero, inspirado en la teoría de los actos de habla de Searle (1969), se basa en la especificación de una condición esencial para los actos con fuerza insultiva, en analogía con la condición esencial especificada para otros tipos de actos, tales como los que poseen fuerza compromisiva. El segundo argumento, inspirado en ciertas ideas de Strawson (1964), muestra que la intención peyorativa, esencial a los usos insultivos, es un tipo de intención comunicativa y, por tanto, se la puede considerar indicativa de una fuerza ilocucionaria específica.

2.2. Primer argumento: la presencia de una intención peyorativa como condición esencial del acto de insultar

En su intento por especificar la estructura de los actos ilocucionarios, Searle dice en relación con el acto de prometer:

7. H intenta que la emisión de $\mathrm{T}$ lo coloque a él bajo la obligación de hacer A. La característica esencial de una promesa consiste en asumir la obligación de realizar un cierto acto. Pienso que esta condición distingue a las promesas (y a otros miembros de la misma familia, tales como los votos) de otros géneros de actos ilocucionarios. Obsérvese que en el enunciado de la condición solamente se especifica la intención del hablante; otras condiciones adicionales clarificarán cómo ha de entenderse esa intención. [...] Llamo a esto la condición esencial. (1969, p. 68; las cursivas son nuestras.)

De ella deriva lo que llama "la regla esencial" para el acto de prometer, según la cual "la emisión de $\operatorname{Pr}$ cuenta como la asunción de una obligación de hacer A” (p. 71). En analogía con este análisis, proponemos la siguiente condición esencial para el acto de insultar a alguien mediante un término peyorativo de grupo:

(15) Dado un hablante $H$ que emite una oración $T$ que incluye el término peyorativo de grupo " $g$ " frente a un oyente $O$, al emitir $T, H$ insulta a $O$ si y sólo si $H$ intenta que la emisión de $T$ exprese su desprecio por el grupo $G$ que incluye a $O$, es decir, $H$ intenta que la emisión de $T$ constituya un respaldo activo del estereotipo semántico de " $g$ ". 
Asimismo, derivamos la siguiente regla esencial: el acto de insultar a alguien por medio de un peyorativo de grupo cuenta como la expresión de desprecio hacia los miembros del grupo al que la persona insultada pertenece, esto es, el respaldo activo del estereotipo semántico del peyorativo. De este modo, lo esencial a este sub-tipo de acto de insultar es que el hablante tenga lo que antes caracterizamos como una intención peyorativa dirigida hacia los miembros del grupo a los que se aplica el término. ${ }^{21}$

De acuerdo con esta propuesta, un uso de (16)

(16) Eres (un) sudaca: por eso no has sido invitado a mi fiesta.

es un insulto expresivamente correcto aplicado al interlocutor si y sólo si, no solo es emitida en un contexto en el que está vigente un estereotipo cultural asociado con los sudamericanos, condensado en el estereotipo semántico de "sudaca", sino además el hablante tiene una intención peyorativa hacia los miembros de ese grupo en el que incluye a su interlocutor. Retomando el ejemplo inicial, mientras que todo uso de (1) tiene como condición de corrección expresiva el que el estereotipo cultural asociado con los sudamericanos esté vigente en el contexto, un uso específicamente insultivo de esa oración tiene una condición de corrección expresiva más específica: suma a la vigencia del estereotipo cultural en cuestión, la presencia en el hablante de una intención peyorativa dirigida hacia el grupo de referencia:

(17) "Diego es sudaca" es un insulto expresivamente correcto aplicado a la persona referida por "Diego" si y sólo si es emitida en un contexto en el que (i) está vigente un estereotipo cultural asociado con los sudamericanos, condensado en el estereotipo semántico de "sudaca", (ii) el hablante tiene una intención peyorativa hacia los miembros de ese grupo en el que incluye a esa persona.

La única diferencia entre (1) y (16) es que al emitir (1) con una intención peyorativa el insulto se aplica o tiene por objeto a una tercera persona, mientras que al emitir (16), se aplica o tiene por objeto al oyente o interlocutor.

Ahora bien, así como en el caso de las promesas la presencia del verbo "prometer" es considerada uno de los dispositivos indicadores

${ }^{21}$ Decimos "sub-tipo" porque, como se recordará, también es posible insultar mediante el uso de otro tipo de expresiones peyorativas y de expresiones puramente descriptivas. 
de fuerza ilocucionaria compromisiva, es posible preguntarse cuáles serían los dispositivos indicadores de fuerza ilocucionaria insultiva. Creemos que son fundamentalmente elementos que determinan no el contenido proposicional de la emisión sino lo que llamaremos su "carga evaluativa negativa", la cual está constituida por el respaldo activo del estereotipo correspondiente por parte del hablante, y, en particular, por su suscripción, mediante la adopción de una actitud evaluativa individual, de la valencia negativa que lo caracteriza. Como dijimos en la sección anterior, el estereotipo asociado con un peyorativo de grupo, además de incluir un conjunto de rasgos, posee una valencia negativa. En los usos insultivos, el hablante suscribe ese valor, adoptando una actitud evaluativa del mismo signo hacia los miembros del grupo de referencia: esto da a la emisión en juego una carga evaluativa negativa — esto es, además de tener un valor de verdad desde el punto de vista condicional-veritativo, desde el punto de vista expresivo, está negativamente cargada—. ${ }^{22}$ En términos del ejemplo anterior, diremos que un uso insultivo de (1) es verdadero si y sólo si Diego es sudamericano y tiene una carga evaluativa negativa si y sólo si el hablante suscribe, mediante su respaldo activo del estereotipo de "sudaca", la valencia negativa correspondiente, es decir, evalúa negativamente al grupo de referencia. De este modo, una emisión con fuerza insultiva no sólo tiene una condición de verdad que al darse efectivamente determina su verdad, sino que también tiene una condición de corrección insultiva que al darse efectivamente determina su carga evaluativa negativa. ${ }^{23}$

${ }^{22}$ Podría decirse que la carga evaluativa es a la dimensión expresiva de una emisión lo que el valor de verdad es a su dimensión representacional.

${ }^{23}$ Esto nos permite responder, aunque sea en parte, una objeción importante realizada por un evaluador anónimo: la fuerza ilocucionaria insultiva no parece poder hacerse explícita a través del uso de un verbo, como es el caso, por ejemplo, de la fuerza compromisiva que se hace explícita a través del uso del verbo "prometer" en emisiones del tipo de "En virtud de este acto, prometo que llegaré temprano", equivalente, en cierto contexto a la emisión de "Llegaré temprano". De acuerdo con lo dicho en el texto, consideramos que la fuerza ilocucionaria insultiva de una emisión con un peyorativo de grupo se hace explícita a través no de un elemento determinante del contenido proposicional de una supuesta emisión equivalente (un cierto verbo) sino de un elemento que determina la carga evaluativa negativa de la emisión original, a saber, el respaldo o la suscripción, por parte del hablante, de la valencia negativa del estereotipo asociado con el peyorativo correspondiente (mediante la adopción de una actitud evaluativa individual del mismo signo). Desde este punto de vista, todo insulto realizado mediante el uso de un peyorativo de grupo sería explícito. 
2.3. Segundo argumento: la intención peyorativa como intención comunicativa

Como anticipamos previamente, en este apartado nos basaremos en Strawson (1964) para establecer que (i) al insultar a un oyente mediante un término peyorativo de grupo el hablante tiene la intención de generarle un estado mental - la captación de su desprecio por el grupo de referencia en el que se lo incluye - mediante el reconocimiento de su intención de generárselo, es decir, la intención peyorativa que identificamos como esencial al acto de insultar es una intención comunicativa; por consiguiente, se trata de un acto ilocucionario; (ii) el acto de insultar, como todo acto de habla complejo, involucra distintas intenciones comunicativas, aunque la intención peyorativa sea la más importante; (iii) el acto ilocucionario de insultar puede distinguirse del acto perlocucionario de ofender. La siguiente cita es útil para ilustrar todos los puntos anteriores:

Así pues en el caso en el que no solamente te informo sino que también te advierto que $p$, entre las intenciones que intento que reconozcas [...] están no sólo conseguir tu creencia de que $p$, sino también la intención de conseguir que tú estés sobre aviso respecto de los peligros de $p$. La diferencia entre jactarse y advertir es que tu reconocimiento de mi intención de ponerte en guardia puede muy bien contribuir a ponerte en guardia mientras que tu reconocimiento de mi intención de impresionarte no contribuye igualmente a mi acción de impresionarte [... ] (1964, p. 186; las cursivas son nuestras.)

En primer lugar, como está claro en la cita, el acto de advertir es un acto de habla complejo: hay más de un efecto que el hablante intenta producir en el oyente al realizar una advertencia. Por un lado, tiene la intención de informarlo acerca de cierta situación en el mundo - esto es, de generarle la creencia de que el mundo es de determinada manera, por ejemplo, que hay un incendio en un lugar cercano al oyente - y, además, tiene la intención, más importante, de advertirle que está en peligro — esto es, de generarle la intención de ponerse a salvo o en guardia- . De este modo, podría decirse que el acto de advertir es implementado por un acto de habla más básico, una aserción, esto es, un acto de habla con fuerza ilocucionaria asertiva: al afirmar que hay un incendio en un lugar cercano al oyente, el hablante le advierte que está en peligro. En este punto cabe hacerse la siguiente pregunta metafísica: ¿hay aquí dos actos distintos o un solo acto que puede ser descripto de dos maneras alternativas? Sin pretender solucionar el problema ontológico, sólo diremos, en 
primer lugar, que se trata de un problema general, no específico de los actos de habla. Como señala Davidson (1980), el problema es ubicuo: ¿̨cuando alguien enciende la luz y por eso alerta al ladrón está realizando dos acciones distintas o una sola que puede ser descripta de dos maneras alternativas? En segundo lugar, sugeriremos, en la línea davidsoniana, que, dado que la intención de poner al otro en guardia es más importante que la intención de informarlo, la descripción más acabada y específica del único acto de habla involucrado es la que lo presenta como una advertencia, mientras que la que lo presenta como una aserción es una descripción en términos más básicos.

Ahora bien, desde nuestra perspectiva, al insultar, como al advertir, hay un efecto adicional que el hablante intenta producir en el oyente: también en este caso el hablante busca una respuesta primaria compleja. Así, cuando el hablante emite (16), repetida aquí para facilitar la lectura,

(16) Eres (un) sudaca: por eso no has sido invitado a mi fiesta.

tiene, por un lado, la intención de informar al oyente acerca de cierta situación en el mundo, esto es, generarle la creencia de que pertenece al grupo de los sudamericanos con el que se asocian ciertos rasgos estereotípicos con valencia negativa (intención clasificatoria); pero, además, tiene la intención, más importante, de expresarle su desprecio por el grupo de referencia, es decir, generarle la captación de su actitud emocional (intención peyorativa). Por lo tanto, el acto de insultar es implementado por un acto de habla más básico, una aserción, esto es, un acto de habla con fuerza ilocucionaria asertiva: al afirmar que su interlocutor es (un) sudaca, el hablante está insultándolo. Pero, nuevamente, dado que la intención peyorativa es más importante que la intención informativa, diremos que la descripción más acabada y específica del único acto de habla involucrado es la que lo presenta como un insulto, es decir, un acto con fuerza ilocucionaria insultiva, mientras que la que lo presenta como una aserción es una descripción en términos más básicos.

En segundo lugar, queremos destacar la distinción que Strawson realiza entre el acto de advertir y el acto de jactarse o impresionar: el primero logra su efecto mediante un procedimiento griceano - el oyente se pone en guardia porque reconoce la intención del hablante de ponerlo en guardia-, mientras que el segundo no logra su efecto de ese modo - el oyente no se impresiona por reconocer la intención del hablante de impresionarlo- - . En el texto al que pertenece 
la cita anterior, el autor propone considerar el tipo de procedimiento involucrado en la producción de efectos como un criterio para distinguir entre actos ilocucionarios y actos perlocucionarios: si el procedimiento es griceano, el acto es ilocucionario mientras que si es de cualquier otro tipo, el acto es perlocucionario. De este modo, concluye que advertir es un acto ilocucionario mientras que jactarse o impresionar es un acto perlocucionario.

Pues bien, desde nuestra perspectiva, insultar es como advertir y no como impresionar, y, por tanto, constituye un acto ilocucionario. Al emitir (16), el hablante no sólo intenta que su interlocutor reconozca su intención de generarle la creencia de que pertenece al grupo de los sudamericanos con el que se asocian ciertos rasgos estereotípicos con valencia negativa, es decir, su intención clasificatoria, sino que también intenta que reconozca su intención de expresarle su desprecio por el grupo en cuestión, esto es, su intención peyorativa. El reconocimiento por parte del oyente de la intención del hablante de expresarle su desprecio por los sudamericanos es lo que contribuye a que esa intención se cumpla, es decir, a que el oyente capte ese desprecio. Tómese en cuenta que la sola comprensión de "sudaca" no es suficiente para producir ese efecto, puesto que la actitud emocional del desprecio no es parte del significado expresivo del término sino un factor expresivo de tipo pragmático. En general, cuando el hablante insulta a alguien mediante un peyorativo de grupo no solamente intenta producir en el oyente una determinada actitud proposicional (la creencia de que la persona insultada, sea ésta el mismo oyente o una tercera persona, pertenece al grupo de referencia, estereotípicamente caracterizado) sino que también

(i) intenta que el oyente capte su desprecio por el grupo de referencia, esto es, capte una determinada actitud emocional;

(ii) intenta que el oyente reconozca su intención (i);

(iii) intenta que sea el reconocimiento por parte del oyente de su intención (i) lo que le proporcione a éste una razón para captar su desprecio.

Como podrá apreciarse, las condiciones (i), (ii) y (iii) constituyen los requisitos griceanos para considerar que estamos en presencia de una intención comunicativa. Por consiguiente, de acuerdo con el criterio que Strawson sugiere, en la medida en que el acto de insultar involucra un procedimiento griceano, se trata de un acto 
ilocucionario, esto es, hay actos de habla con fuerza ilocucionaria insultiva. $^{24}$

Finalmente, la intención peyorativa puede distinguirse de otra intención también presente o, en otras palabras, de otro efecto que el hablante puede intentar producir en el oyente al insultarlo: ofenderlo, hacer que se sienta herido en su autoestima. ${ }^{25}$ Desde nuestra perspectiva, se trata de un efecto perlocucionario, que no es parte esencial del acto de insultar. ${ }^{26}$

La ofensa no es un efecto que el hablante intenta que se produzca por medio del reconocimiento de su intención de ofender pues tal reconocimiento puede dejar al oyente totalmente indiferente, es decir, es compatible con que el oyente no se ofenda en absoluto. En términos del ejemplo anterior, un oyente que, ante la emisión de (16), capta el desprecio del hablante por los sudamericanos, grupo en el que lo incluye, puede no sentirse herido en su autoestima, por ejemplo, por considerar que la creencia en cuestión es moralmente incorrecta y porque el desprecio del hablante por los sudamericanos sólo le genera su propio desprecio por el hablante. En general, ofender parece ser como impresionar, pero no en el sentido de que el reconocimiento de la intención de quien se jacta produce el efecto contrario sino porque el reconocimiento de la intención de ofender no es suficiente para que alguien se sienta ofendido: si el oyente carece de cierta sensibilidad o predisposición de carácter o si posee creencias morales lo suficientemente fuertes como para desacreditar las actitudes moralmente reprobables del hablante, reconocer la intención en cuestión lo dejará completamente indiferente.

De este modo, así como Strawson opone el acto ilocucionario de advertir al acto perlocucionario de jactarse o impresionar, oponemos

${ }^{24}$ Un evaluador anónimo ha presentado la siguiente objeción: intuitivamente parecería que hay casos en los que el oyente no reconoce la intención peyorativa del hablante y, sin embargo, el insulto tiene lugar de todos modos; en términos cercanos a los de Neu (2007), parece posible insultar a alguien sin que esta persona se haga eco del insulto. Acordamos plenamente con esta observación, la cual es compatible con la tesis, defendida en el texto, según la cual la persona que insulta tiene una intención peyorativa que responde a las condiciones de Grice (es decir, es una intención comunicativa). El hecho de que haya casos en los que la intención en cuestión no se cumple no implica que su presencia (aun como intención incumplida) no sea esencial para que el insulto tenga lugar. Nuestra idea es que si nunca se cumpliera, no existiría la práctica del insulto.

${ }^{25}$ Tómese en cuenta que el oyente puede ofenderse por ser objeto de un insulto o, si el insulto está dirigido a una tercera persona, por sentirse afectivamente cercano a la persona insultada - piénsese en un uso insultivo de (1) emitida frente a un amigo de Diego.

${ }^{26}$ Maffía (2007, p. 18) sugiere una distinción semejante. 
el acto ilocucionario de insultar al acto perlocucionario de ofender. Mientras que para que el oyente sea insultado es suficiente con que reconozca la intención peyorativa del hablante, para que se ofenda no es suficiente con que reconozca su intención de ofenderlo. Así, el insulto involucra un procedimiento griceano: el reconocimiento por parte del oyente de la intención del hablante de producir en él determinado efecto (insultarlo) es suficiente para que ese efecto se produzca (sea insultado); la ofensa, en cambio, no funciona de esa manera: el reconocimiento por parte del oyente de la intención del hablante de producir en él determinado efecto (ofenderlo) no es suficiente para que éste tenga lugar (se ofenda). En otras palabras, si un hablante usa un peyorativo de grupo con la intención, reconocida por el oyente, de expresar su desprecio por el grupo de referencia en el que lo incluye, éste es sin duda insultado, pero puede no ofenderse en la medida en que su naturaleza afectiva o su carácter podrían hacerlo inmune al insulto. A menos que el oyente sea sensible a ciertas actitudes y prácticas, aun cuando reconozca la intención peyorativa del hablante, podría permanecer completamente indiferente. Por otro lado, si tuviera esa sensibilidad, podría ofenderse aun cuando el hablante no hubiera tenido ninguna intención peyorativa (y a fortiori, él no hubiera podido reconocer ninguna intención de ese tipo en el hablante).

\section{Conclusión}

En la primera parte del trabajo hemos defendido la tesis de que, desde el punto de vista semántico, los términos peyorativos de grupo poseen dos factores: (i) un significado representacional idéntico al de sus respectivas contrapartes neutras, y (ii) un significado expresivo constituido por un estereotipo de carácter evaluativo, a saber, una lista abierta de conceptos descriptivos, densos y puramente evaluativos que codifica un valor global negativo, su valencia característica. El estereotipo es entonces un componente del significado del término, aquello que el hablante está obligado a captar para aprender a usarlo, para ser competente - aunque, dado su carácter abierto, cada hablante puede captar una parte distinta del estereotipo-. Además, a diferencia del sentido fregeano y de la intensión en sentido tradicional, el estereotipo no tiene la función semántica de determinar la extensión del término sino que es completamente independiente de aquélla. En la segunda parte, hemos argumentado en favor de la tesis de que hay otro componente expresivo que es aportado por el uso original y paradigmático de los términos peyorativos de grupo, 
al que caracterizamos como insultivo: aquel en el que el hablante tiene una intención peyorativa dirigida a los miembros del grupo de referencia. Este segundo componente expresivo no es semántico sino pragmático, es decir, no es parte del significado del término, pero está estrechamente relacionado con él: involucra un respaldo activo del estereotipo y, por tanto, una reactivación, con peso político, de la visión prejuiciosa que éste revela, condensa y estabiliza. ${ }^{27}$

\section{BIBLIOGRAFÍA}

Anderson, L. y E. Lepore, 2013a, "Slurring Words", Noûs, vol. 47, no. 1, pp. 25-48.

— 2013b, "What Did You Call Me? Slurs as Prohibited Words", Analytic Philosophy, vol. 54, no. 3, pp. 350-363.

Austin, J.L., 1962, How to Do Things with Words, Clarendon Press, Oxford.

Blackburn, S., 1992, "Morality and Thick Concepts: Through Thick and Thin", Proceedings of the Aristotelian Society, supplementary vol., no. 66, pp. 285-299.

Camp, E., 2013, "Slurring Perspectives", Analytic Philosophy, vol. 54, no. 3, pp. 330-349.

Caso, R. y N. Lo Guercio, 2016, "What Bigots Do Say: A Reply to DiFranco", Thought: A Journal of Philosophy, vol. 5, no. 4, pp. 265274.

Davidson, D., 1980, Essays on Actions and Events, Oxford University Press, Oxford.

Gibbard, A., 1992, "Morality and Thick Concepts: Thick Concepts and Warrant for Feelings", Proceedings of the Aristotelian Society, supplementary vol., no. 66, pp. 267-283.

Grice, H.P., 1967, "Logic and Conversation", en Studies in the Way of Words, Harvard University Press, Cambridge.

Gutzmann, D., 2015, Use-Conditional Meaning. Studies in Multidimensional Semantics, Oxford University Press, Oxford.

Hom, Ch., 2010, "Pejoratives", Philosophy Compass, vol. 5, no. 2, pp. 164185.

${ }^{27}$ Este trabajo fue escrito en el marco de los subsidios "Predicados normativos: de los enunciados de gusto a los juicios estéticos" (UBACyT 20020170100649BA, Res. 1041/2018), otorgado por la Universidad de Buenos Aires, y "Deflacionismo, correspondentismo y hacedores de verdad" (PICT 2016-0438), otorgado por la Agencia de Promoción Científica y Tecnológica. Agradecemos a los miembros de BA-LingPhil, especialmente a Ramiro Caso, Eduardo García Ramírez, Nicolás LoGuercio, Alfonso Losada y Laura Skerk, así como a María de Ponte, Kepa Korta, Alberto Moretti, Federico Penelas, Diana Pérez, Marco Ruffino y Ludovic Soutif, por sus comentarios a versiones previas de este trabajo. 
Hom, Ch., 2008, "The Semantics of Racial Epithets", The Journal of Philosophy, vol. 105, no. 8, pp. 416-440.

Jeshion, R., 2013a, "Slurs and Stereotypes", Analytic Philosophy, vol. 54, no. 3, pp. 314-325.

- 2013b, "Expressivism and the Offensiveness of Slurs", Philosophical Perspectives, vol. 27, no. 1, pp. 231-259.

Kaplan, D., 1999, “The Meaning of Ouch and Oops: Explorations in the Theory of Meaning as Use", MS, University of California, Los Ángeles.

Kirchin, S., 2017, Thick Evaluation, Oxford University Press, Oxford.

Losada, A., 2017, "Sudaca. A Semantics for Slurs", MS.

Maffía, D., 2007, "Epistemología feminista: la subversión semiótica de las mujeres en la ciencia", Revista Venezolana de Estudios de la Mujer, vol. 12, no. 28, pp. 63-97.

McCready, E., 2010, "Varieties of Conventional Implicature", Semantics and Pragmatics, vol. 3, no. 8, pp. 1-57.

McDowell, J., 1981, "Non-Cognitivism and Rule-Following", en Stephen Holtzman y Christopher Leich (comps.), Wittgenstein: To Follow a Rule, Routledge and Kegan Paul, Londres.

Neu, J., 2007, Sticks and Stones: the Philosophy of Insults, Oxford University Press, Oxford.

Nunberg, G., 2016, "The Social Life of Slurs", en D. Fogal, D. Harris y M. Moss (comps.), New Work on Speech Acts, Oxford University Press, Oxford.

Potts, C., 2005, The Logic of Conventional Implicatures, Oxford University Press, Oxford.

—_, 2003, "Expressive Content as Conventional Implicature", en Makoto Kadowaki y Shigeto Kawahara (comps.), Proceedings of the North East Linguistic Society, vol. 33, pp. 303-322.

Predelli, S., 2013, Meaning without Truth, Oxford University Press, Oxford.

Putnam, H., 1975, "The Meaning of 'Meaning'", en Mind, Language and Reality. Philosophical Papers, vol. 2, Cambridge University Press, Cambridge.

—_, 1970, “Is Semantics Possible?", en Mind, Language and Reality. Philosophical Papers, vol. 2, Cambridge University Press, Cambridge.

Schlenker, Ph., 2007, "Expressive Presuppositions", Theoretical Linguistics, vol. 33, no. 2, pp. 237-245.

Searle, J., 1969, Actos de habla. Ensayo de filosofía del lenguaje, trad. L. Valdés Villanueva, Cátedra, Madrid.

Strawson, P., 1964, "Intención y convención en los actos de habla", en Ensayos lógico-lingüisticos, trad. L. Valdés Villanueva y A. García Suárez, Tecnos, Madrid.

Tirrell, L., 1999, "Derogatory Terms. Racism, Sexism, and the Inferential Role Theory of Meaning", en Christina Hendricks y Kelly Oliver 
(comps.), Language and Liberation. Feminism, Philosophy, and Language, State University of New York Press, Albania, pp. 41-79.

Whiting, D., 2013, “It's Not What You Said, It's the Way You Said It: Slurs and Conventional Implicature", Analytic Philosophy, vol. 54, no. 3, pp. $364-377$.

Williams, B., 1985, Ethics and the Limits of Philosophy, Harvard University Press, Cambridge.

Wittgenstein, L., 1953, Investigaciones filosóficas, trad. A. García Suárez y U. Moulines, Crítica, Barcelona.

Recibido el 24 de mayo de 2019; revisado el 14 de octubre de 2019; aceptado el 24 de octubre de 2019. 